\title{
Ethnologies
}

\section{Tourisme et migration dans le Maramureş}

\section{Raluca Nagy}

Volume 31, numéro 1, 2009

\section{Passages}

URI : https://id.erudit.org/iderudit/038502ar

DOI : https://doi.org/10.7202/038502ar

Aller au sommaire du numéro

Éditeur(s)

Association Canadienne d'Ethnologie et de Folklore

ISSN

1481-5974 (imprimé)

1708-0401 (numérique)

Découvrir la revue

Citer cet article

Nagy, R. (2009). Tourisme et migration dans le Maramureş. Ethnologies, 31(1), 111-126. https://doi.org/10.7202/038502ar

\section{Résumé de l'article}

Dans cet article nous entreprenons de souligner la liaison entre deux formes de mobilité, tourisme rural et migration de travail, à travers quelques points d'articulation. Dans le cas du Maramures, une région du nord de la Roumanie, on se trouve devant deux types de " couloirs de mobilité ", vers et à partir de la région. Ces couloirs se superposent au niveau des pratiques autant qu'au niveau des réseaux et des ressources. Les cinq points plus concrets qu'on va aborder ici sont la circulation de l'argent, le rôle des médiateurs culturels des migrants, la question du confort et la façon d'investir dans les maisons, la problématique de la latinité et finalement l'angle terminologique, notamment le continuum tourisme - migration.
Ce document est protégé par la loi sur le droit d'auteur. L'utilisation des services d'Érudit (y compris la reproduction) est assujettie à sa politique d'utilisation que vous pouvez consulter en ligne.

https://apropos.erudit.org/fr/usagers/politique-dutilisation/ 


\title{
Tourisme et migration dans le Maramureş
}

\author{
Raluca Nagy \\ Université Libre de Bruxelles \\ École Nationale d'Études Politiques et Administratives, Bucarest
}

Mon intérêt pour le Maramureş, une région du Nord de la Roumanie, se concentre sur le changement que des formes de mobilité comme le tourisme et la migration ont apporté à la relation entre tradition et modernité. L'appréhension lucrative de l'archaïque et de l'authenticité, des entrées d'argent qui transforment en partie les activités économiques traditionnelles, des changements sociaux et des représentations, voici quelques effets "classiques"du tourisme. En même temps, à partir de ma toute première recherche de terrain ${ }^{1}$, il apparaît que la migration de travail des villageois à l'étranger est une réalité de tous les villages (mais pas exclusivement) de l'Europe de l'Est et s'avère un phénomène très présent.

À un niveau superficiel, il y a déjà des ressemblances entre tourisme et migration en tant que formes de mobilité : les deux impliquent une région de départ et une région d'accueil (souvent la même) et les deux

1. Les données nécessaires pour cette analyse font partie d'une recherche doctorale intitulée "La Marchandisation des traditions. Étude de cas dans le Maramureş en Roumanie ", sous la double direction de Vintila Mihailescu à l'École Nationale d'Études Politiques et Administratives de Bucarest et de Marianne Mesnil à l'Université Libre de Bruxelles. La recherche de terrain s'est déroulée entre 2003 et 2006 dans des villages situés autour du mont Gutai, dans la Valée d'Iza et celle de Viseu, c'est-à-dire dans le Maramureş dit " historique ". Je me suis rendue cinq fois sur le terrain pendant environ un mois en couvrant toutes les saisons de l'année pour avoir un aperçu aussi complet que possible du phénomène touristique. 
représentent un voyage et un détachement de chez soi. Les groupes sociaux locaux, comme les familles ou les réseaux de connaissances, sont très importants pour le contrôle de l'accès au tourisme rural et à la migration, conduisant à l'inclusion et exclusion des acteurs de ce phénomène.

Dans cet article, je vais d'abord faire une brève présentation de la perspective concernant les différentes formes de mobilité, sur laquelle je voudrais baser mon analyse. J'exposerai ensuite un très bref historique de la région, avant de décrire la spécificité du tourisme rural et de la migration au Maramureş, pour arriver enfin à dégager quatre exemples d'articulation.

\section{Le continuum² tourisme - migration}

Ce qu'on reproche souvent aux études anthropologiques touchant au tourisme c'est d'échouer "à faire la distinction entre les conséquences sociales du tourisme et d'autres processus de changement qui ont lieu indépendamment dans une société " (Boissevain 1977 ; Crick 1989) ${ }^{3}$. Autrement dit, les influences du tourisme sont rarement distinguées d'autres forces contemporaines de changement social.

Éviter le piège de l' "égocentrisme » touristique s'inscrit dans un effort assez récent dans les sciences sociales pour aborder la diversité des formes de mobilité de nos jours. Le besoin d'une approche critique aux problèmes de la mobilité et de l'appartenance devient de plus en plus pressant. Un nombre grandissant d'auteurs (Chapman et Prothero 1985; Appadurai 2005; O'Reilly 2003; Williams et Hall 2002; Lenz 2006 ; Romhild 2003) considèrent que les nouvelles formes de mobilité remettent en question la distinction nette entre tourisme et migration et s'interrogent sur la pertinence d'une analyse séparée de ces deux notions. L'analyse de la mobilité humaine a besoin de nouvelles conceptualisations et surtout de nouvelles méthodologies.

Certains auteurs parlent de "circulation" (Chapman et Prothero 1985); ceux qui ne s'accordent pas sur une représentation circulaire du mouvement, puisque celle-ci envisage un retour au point de départ, parlent de «mouvement " ou de "déplacement temporaire " (Williams et Hall 2002). Quant à la dénomination des participants à ces mobilités,

2. Voir O'Reilly 2003.

3. Crick nous parle aussi des cinq " $s$ " du tourisme : sun, sex, sights, savings and servility (1982). 
l'on s'accorde généralement sur "personnes qui s'installent ", plutôt que " touristes» ou « migrants». Ceci s'explique principalement par le fait que l' "installation » serait la ressemblance principale entre tourisme et migration: ces deux phénomènes représentent une forme d'établissement ou d'installation temporaire dans un endroit différent que celui d'origine (Romhild 2003).

Certaines analyses critiques de la littérature existante sur le sujet (Abram et Waldren 1997 ; Braunlein et Lauser 1997) nous montrent que les études sur le tourisme ainsi que sur la migration avaient tendance à s'attacher à un idéal sédentaire, en allant contre leurs acteurs principaux : les gens qui voyagent. Le tourisme était une fuite temporaire de la vie quotidienne et la migration, un changement souvent irréversible.

La nouveauté de la recherche actuelle, en partie liée aux processus de globalisation, indique l'abandon de ces paradigmes sédentaires et la compréhension des mobilités comme des projets (de vie), des processus qui font la liaison entre des espaces et permettent des connections multiples. De plus, étant donné que les destinations ainsi que les points de départ touristiques et migratoires sont souvent les mêmes, cette perspective peut nous aider à visualiser les ressemblances et les liaisons entre ces différentes formes de mobilité et pourrait nous être plus utile que de percevoir ces dernières en tant que phénomènes sociaux séparés. L'avantage principal des métaphores modernes du mouvement est qu'elles nous aident à problématiser les phénomènes culturels en termes de processus (O'Reilly 2003).

Les études sur la migration conceptualisent de plus en plus la migration de type circulaire / saisonnière / oscillatoire. D'après Williams et Hall, le tourisme même constitue une forme de migration ou la migration, une forme de tourisme. Les auteurs iront jusqu'à nous proposer un circuit séquentiel d'interdépendance entre les deux. Le tourisme est souvent défini en termes de ce qu'il n'est pas, ou comme une inversion du cours normal des choses (Urry 2000). Néanmoins, l'expérience des migrants eux-mêmes vers leur pays d'origine entre parfaitement dans ce genre de définition. L'analyse des pratiques touristiques nous permet de constater que celles-ci sont souvent reprises par les migrants et inversement. Si l'on observe le comportement des migrants quand ils débarquent dans leurs villages de départ pendant l'été ou les fêtes d'hiver, on voit des touristes: des gens qui débarquent en famille, s'installant dans les maisons, faisant la fête et déambulant au centre du village. En 
fait, tous ces gens prennent leurs deux ou trois semaines de congé ; ils font des promenades dans la région, des visites, participent aux fêtes, ce qui fait aussi partie du parcours des touristes.

Mais en dehors de toutes ces ressemblances, il est encore plus intéressant pour une recherche anthropologique de s'intéresser aux assemblages de pratiques et de stratégies touristiques et migratoires, à la transformation du capital culturel en capital économique et viceversa et à la représentation des acteurs par rapport à ces formes de mobilité qui génèrent ressemblance et rejet, échange et refus, mélange et ségrégation.

\section{Historique et discours sur la région}

La position géographique et l'importance stratégique de la région, doublées par la réalité historique, ont fait du Maramureş un endroit assez isolé, avec une autonomie interne importante. À cette autonomie parfois plus officieuse qu'officielle, s'ajoute la situation géographique : le Maramureş est une zone de frontière. Allant plutôt contre la tendance "nationaliste ", constructrice de l'État roumain de l'époque, H.H. Stahl propose un concept intéressant pour aborder la complexité de cette région frontalière qu'est le Maramureş. Il insiste notamment sur le rôle qu'elle joue dans le cadre des régions élargies en décrivant l'« effet de culasse ", c'est-à-dire un soulignement des traditions comme forme de justification de l'authenticité et de résistance culturelle. Pour le Maramureş historique, dont un tiers se trouve en Roumanie et les deux autres en Ukraine, les changements de population, soit à cause de l'appartenance géopolitique, soit à cause de sa position frontalière, n'ont fait que renforcer la tradition locale. Cette authenticité, préservée grâce à sa situation géographique, pourrait aussi s'expliquer par la caractéristique de "zone ethnographique archaïque "(Rey 2002) : la position dans une région montagneuse la rend difficilement accessible et en dehors des grandes routes commerciales, elle est donc moins touchée par les diverses influences.

En vertu de ces caractéristiques, le Maramureş se trouve parmi les régions qui ont permis d'asseoir les discours de la construction de l'État national basés sur le "paysan parfait " et l'image du berceau d'authenticité. On assiste à un discours invariable sur l'authenticité du Maramureş, discours repris pendant le communisme avec toutes sortes de contradictions et de dissimulations ; pour promouvoir "l'homme nouveau ", l'ouvrier, on avait besoin du paysan. 
Après 1990, c'est le tourisme qui a repris le discours sur la tradition et l'authenticité. Ce modèle «villageois», hors du temps, imaginé dans le temps universel, totalement idéalisé, se prête parfaitement à la promotion touristique. Les gens veulent voir cette société qui constitue les derniers signes visibles de l'état archaïque du monde; en échange, une telle société répond à ces attentes et demandes d'authenticité par une mise en scène des traditions.

Le tourisme rural et les relations habltant - visiteur

Dans la grande majorité des cas, la présence des étrangers implique pour les membres d'une société locale la mise en scène de leur culture et l'adoption du "rôle ethnique ». Pour les sociétés " touristifiées » et surtout dans le cas du tourisme rural, il n'est pas sûr que ce rôle prenne une dimension essentielle et qu'il se substitue à toutes les autres identités, mais il prend nécessairement une place plus importante qu'ailleurs.

De la littérature parcourue, on peut retenir trois manières de réagir à cette augmentation du rôle identitaire par rapport à l'intégration de l'intérêt "traditionnel " externe dans l'identité des habitants. Dans un premier scénario, la société locale peut répondre à l'image qu'on lui assigne sans renoncer à sa diversité culturelle. Le recours à l'authenticité culturelle ne constituerait qu'une "ressource mobilisable en temps utile, lorsqu'il est avantageux pour la société de promouvoir son caractère traditionnel " (Doquet $2001: 8$ ). On assiste ici à une illusion de tradition; autrement dit, le Maramuresean sait très bien comment se passent les choses derrière sa porte et dans sa vie mais pour les différents curieux qui viennent le voir, il joue le paysan archétypal du XIX' siècle.

Une deuxième réaction possible est le faux archaïsme (Rosenow et Pulsipher 1979) qui suggère que les gens sur place ont déjà renoncé à l'authenticité culturelle plus que le spectacle et la mise en scène ne le laissent ressortir. Ce faux archaïsme est plus transparent et se présente comme une combinaison bizarre entre traditionnel et moderne que les visiteurs catégorisent souvent de "kitsch ": les façades des maisons en faïence à la place de la vieille mosaïque en couleurs, les clôtures "léopard ", les canapés en cuir, la horinca (alcool local très fort) vendue dans des bouteilles de Coca-Cola, les icônes, les chaînes et autres objets traditionnels en plastique.

À l'autre extrême de l'illusion de la tradition se trouve le troisième scénario possible, où le rôle ethnique pourrait se prolonger dans 
différentes interactions, même en l'absence de l'étranger. La répétition d'un rôle identitaire pourrait entraîner une cristallisation de l'identité (Garabuau 1996) et les habitants se prendraient finalement au jeu de ce qu'ils racontent pour le touriste.

J'ai choisi cet angle du "rôle ethnique " parce qu'il permet de centrer la problématique sur la relation entre les touristes et la population locale, au lieu de considérer ces deux groupes séparément. Ces trois manières de réagir s'entrechoquent et se substituent en fonction de plusieurs caractéristiques que l'habitant adapte à chaque situation et en fonction du visiteur. On glisse et on mélange ces trois types de rôle ethnique sur deux niveaux complètement différents et variables en fonction du touriste. Autrement dit, la relation entre habitant et touriste varie de l'extrême "amitié " - être reçu presque comme un ami ou un membre de la famille - au pur "accueil touristique », où le touriste se retrouve complètement dans la logique du contrat. En plus, la relation entre hôte et voyageur peut basculer d'un jour à l'autre, passant du "paquet touristique" (logement et trois repas par jour pour un prix fixe et non négociable), à une attention particulière de la part de l'hôte sans que ce dernier ne demande de rétribution financière. Les voyageurs se divisent tout le temps entre les catégories d' "étrangers" et de "proches". Souvent, les voyageurs sont envoyés par la famille ou des amis et on ne leur réserve pas vraiment le traitement de touriste. Dans ce cas, l'offre ne s'adresse finalement pas au touriste même, mais à celui qui l'a envoyé et c'est ce dernier qui doit être satisfait de l'accueil.

Tout ce processus entraîne une négociation permanente de la relation entre hôte et visiteur et qui peut s'avérer très bouleversante pour ce dernier. Mais cette diversité d'accueil est en faite la preuve que l'on trouve rarement au Maramureş le tourisme en tant qu'activité économique dans le vrai sens du mot: les calculs ne se font pas dans la logique de la rentabilité, du profit ou d'une spécialisation. L'agrotourisme I tourisme rural, pour les gens de Maramureş, n'est pas nécessairement une activité professionnellement construite et ciblée, mais plutôt une improvisation. Cette façon d'improviser nous renvoie à des motivations plus profondes ainsi que contextuelles: le tourisme comme une des stratégies de vie adoptée pendant la période dite "de transition " du socialisme au capitalisme; des auteurs comme Vintila Mihailescu ou Dana Diminescu appellent ces stratégies "pluriactivité " ou "pluriactivité complexe", c'est-à-dire une "multi-stratégie " de vie composée d'activités variées et souvent informelles, trouvant ses racines 
dans le vécu des gens pendant les années du communisme. Dans ce schéma de pluriactivité, le tourisme n'est qu'une des diverses pratiques ou activités possibles. La migration en est une autre.

\section{Les points d'articulation entre tourisme et migration}

La migration de travail ou migration pendulaire est un phénomène relativement nouveau qui a visiblement explosé durant la dernière décennie. "L'effondrement des régimes communistes dans les pays de l'Europe Centrale et Orientale a conduit, entre autres phénomènes, à donner une visibilité à des mouvements de circulation migratoire différents de ceux d'avant 1989 qui étaient pour leur part ancrés dans une longue tradition d'émigration sans possibilité de retour. Les migrations définitives sont devenues rares, tandis que le va-et-vient est bien plus important qu'avant» (Lagrave et Diminescu 1999: 2).

Le Maramureş garde une continuité historique en ce qui concerne la migration de travail. "Les raisons du nomadisme actuel s'inscrivent dans une longue histoire de recherche du travail, puisque les conditions des terres... ne permettent pas, et n'ont jamais permis de vivre de l'agriculture. La mobilité est, en effet, un fait ștructurel de cette région, produit d'une longue habitude historique. L'élevage ovin et le travail forestier ont été deux occupations principales et traditionnelles de cette population.... Ainsi, les paysans ont-ils été contraints à la pluriactivité qui, en l'absence d'autres secteurs d'activités..., les a conduits à une mobilité interne à la région, puis au pays tout entier " (Diminescu 1998: 6). Ce que nous devons retenir de ceci pour comprendre l'articulation entre tourisme et migration, c'est justement cette mobilité structurelle des habitants du Maramureş, de même que leur adaptabilité et leur pluriactivité usuelle.

Pour illustrer le lien concret entre tourisme rural et migration de travail dans le cas du Maramureş, nous allons nous attarder sur quelques points d'articulation, tels que la circulation de l'argent, le rôle des migrants comme médiateurs culturels, la question du confort et la façon d'investir dans les maisons, et pour finir, l'élément de latinité.

\section{La circulation de l'argent}

On peut commencer en affirmant qu'une grosse partie des revenus obtenus par les migrants est indirectement investie dans le tourisme rural et vice-versa. On n'assiste pas à un développement linéaire par les 
entrées d'argent ou les remises de fonds (remittances) comme c'est souvent le cas dans les théories de la migration internationale. Une pratique assez répandue consiste à emprunter deux ou trois mille euros au début de l'été pour quelques petits investissements et achats de produits puis de rembourser cette somme à la fin de la saison; souvent ceux qui fournissent les emprunts sont ceux qui sont partis travailler à l'étranger. Un cas tout aussi fréquent est celui où les migrants envoient de l'argent à leur famille pour payer les achats nécessaires à l'accueil des touristes ou pour investir dans le confort de leurs maisons et construire ainsi par exemple une salle de bain ou une chambre supplémentaire. L'argent gagné grâce aux activités touristiques peut à son tour servir au départ à l'étranger pour trouver du travail : le coût des papiers nécessaires, un premier déplacement, une éventuelle enveloppe pour celui qui se charge de trouver du travail, etc. Parler des montants est toujours une tâche difficile dans cette situation. Au travers les témoignages auxquels j'ai eu accès, ce montant de départ varie entre huit cents et trois mille euros, ce qui, plus que de constituer une somme assez importante, coïncide avec le montant nécessaire pour assurer une saison touristique, comme je l'ai mentionné plus haut.

Les investissements plus importants dans les maisons entrent dans un circuit indirect qui lie tourisme et migration et que je vais expliquer par la suite. Pour l'instant il est important de retenir que les réseaux d'entrées et de sorties d'argent se superposent, ce qui implique que l'inclusion et l'exclusion de la participation aux activités touristiques est souvent liée à la dynamique d'inclusion et d'exclusion de la migration du travail.

\section{Les migrants et leur rôle de médiatelirs culturels}

Un deuxième point d'articulation s'opère à travers les migrants en tant qu'acteurs des deux phénomènes et à travers leur rôle de médiateurs culturels ou passeurs de culture. Les médiateurs "transforment, traduisent, distordent et modifient le sens ou les éléments qu'ils sont censés manipuler" (Latour 2006 : 58). Un cultural broker interprète, traduit et rend accessibles au visiteur les choses qui ne lui sont pas familières, les détails exotiques, étranges et non habituelles, et en faisant cela il a la responsabilité de l'image culturelle reçue. L'expertise dans la communication de la culture est un capital majeur du moment où les touristes sont dépendants pour être connectés aux gens sur place dans les termes de leurs attentes. 
Quand le tourisme a commencé dans les années 1990, le rôle de passeur de culture était "monopolisé » par des entrepreneurs ou des agences de la ville qui entraînaient les villages et les maisons dans leurs tours et circuits organisés. Les " touristés " n'étaient pas très contents de cette situation mais il leur manquait l'expertise et la capacité d'attirer les visiteurs d'une manière régulière. Voici le témoignage d'un habitant par rapport au médiateur culturel avec lequel il collaborait :

Il a la maison là-bas, ils logent chez lui, ils lui payent, il les ramène ici chez nous mais nous, on ne reçoit rien ; j'aimerais les héberger, comme ça qu'est-ce que je gagne, s'ils veulent acheter un chapeau ou un litre de horinca ... je dois arrêter mon travail et m'uccuper d'eux, je dois leur montrer la maisnie $e^{4}$, je ne peux pas dire non, c'est comme un ami qui me demande de lui rendre service. Je vais mettre une affiche: 100.000 lei (trois euros) la visite (G.O., m, 50)!

Quand les choses ont débuté avec plus d'ampleur et que le flux touristique devint plus important, les gens du Maramureş ont appris à jouer leurs propres cultural brokers, les migrants en premier. De plus en plus conscients de leur capital culturel acquis dans le pays d'origine et dans le pays d'accueil, ils ont commencé à s'en servir. Leur premier avantage est temporel et spatial : le retour au pays des migrants correspond aux grosses saisons touristiques (été, hiver et Pâques) en leur permettant de gérer l'accueil directement. À partir de leurs expériences et des contacts personnels avec les étrangers, les migrants ont une expertise plus fine que ceux qui sont culturellement et socialement restreints au contexte local. Une distinction structurelle se forme entre ces migrants qui détiennent l'expertise, le réseau et les ressources nécessaires, et d'autres qui ne possèdent rien de tout cela. Évidemment, être migrant n'est pas une condition nécessaire pour gérer une activité touristique, mais peut souvent faciliter la tâche, si non directement, au moins en transférant l'expertise à la famille qui reste au village. De ce point de vue, les migrants se sentant porteurs de modernité, corrigent et guident le traditionnel sur place. Dans leur communication avec les divers voyageurs, ils ont appris à souligner le mode de vie différent et authentique. "En correspondant à l'attente du touriste, cette image devient de nouveau un lieu de création identitaire" (Romhild 2003 : 11). Ce rôle n'est pour les migrants qu'un des rôles possibles, une façon de montrer leurs compétences, d'avoir le plus d'informations possible sur ces touristes anciens et présents et de gérer

4. Terme proposé par Stahl comme l'équivalent de "gospodarie ». 
leurs attentes. Dans cette médiation culturelle on retrouve souvent une particularité déjà observée : celle de ne pas réfléchir d'une manière exclusivement calculatrice. La capacité à communiquer avec les touristes est d'abord un très bon étalon de statut et de confiance en soi, et elle est un autre moyen de se faire des contacts et des relations, ce qui peut toujours être utile. Loin de se sentir un serviteur des désirs des touristes comme c'est souvent le cas des hôtes, le médiateur est conscient, d'une part, de son statut différent, ensuite, de son pouvoir de choisir ses clients, et finalement, du fait qu'il peut dresser lui-même les conditions et les limites.

\section{La question du confort et les Investissements dans les maisons}

Une caractéristique générale du tourisme rural est le fait que les touristes se plaignent souvent du manque de confort. À l'exception des touristes "fondamentalistes" qui cherchent l'authentique pur et dur, les visiteurs ont certaines exigences en ce qui concerne les conditions pratiques de leur séjour malgré leur désir d'exotisme et d'authentique. Les hôtes doivent offrir à l'étranger le confort dont il a besoin. Ici, il est intéressant d'analyser comment les hôtes ont intégré cette question du confort. À cet égard, les règles du tourisme se reflètent encore plus à travers ce troisième acteur, qui n'est ni hôte ni visiteur, mais plutôt un médiateur culturel et économique : le migrant.

L'impact de la migration sur le tourisme se fait aussi plus indirectement que les entrées directes de liquide ou le «brokerage " culturel. Les Maramureseni anticipent la demande de confort de leurs visiteurs à l'aide d'un feedback qu'ils ont à travers ce qu'ils ont déjà vu a l'étranger ou à travers ce qu'on leur a raconté ou montré. On assiste à une imposition des modèles extérieurs mais souvent avec une double traduction à laquelle s'attache une diversité de réinterprétations locales. Il ne faut pas oublier dans ce contexte l'imitation et l'inspiration.

Maintenant je dois faire la maison d'après les indications que je reçois par téléphone. Nous avons acheté cette maison. Le garçon qui nous l'a vendue n'avait plus l'argent pour la terminer. Mon fils m'a appelé d'Espagne il y a une semaine pour me dire qu'il m'a déjà envoyé l'argent et comment je dois faire les travaux. Je vais faire de mon mieux pour cette maison, pour qu'elle soit moderne et avec les commodités nécessaires. Je ne sais pas exactement ce qu'il veut, mais en gros je sais. Je vois comment sont les maisons que l'on fait maintenant (M.l., m, 53). 


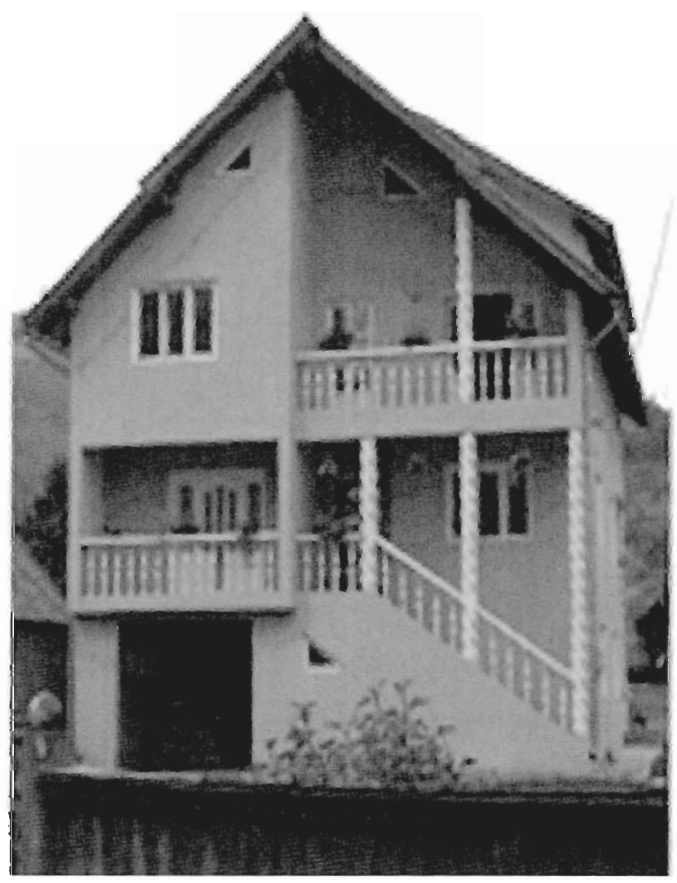

1. Les nouvelles maisons (R. Nagy, 2005).

Souvent les maisons que se font construire ceux qui résident à l'étranger (et qui comptent revenir un jour) servent d'auberge pour les voyageurs. La maison qui reste vide en attendant son propriétaire n'est presque jamais appropriée par la famille qui reste dans le village. Si l'on n'a pas de visiteurs, la maison reste complètement vide. Même quand le propriétaire rentre pour les vacances ou pour son séjour au village entre deux "migrations", il ne loge pas dans la nouvelle maison mais dans l'ancienne.

C'est marrant d'être tous ensemble pour les vacances, on se met tous dans la cuisine et on parle, on parle ... pourquoi déranger toute la nouvelle maison pour quelques semaines ? Tout démarrer, l'eau, le chauffage, c'est trop compliqué. En plus [le migrant] veut se sentir comme au bon vieux temps ou on était tous ensemble à la maison (D.T., migrante, 30 ans). 
L'investissement dans des maisons s'explique tant par l'incapacité d'investir ailleurs que par la volonté d'investir au pays. Ce besoin d'investir n'est pas avoué en tant que tel, mais légitimé au travers du "devoir " social et traditionnel de bâtir une maison pour soi et ses enfants. En parcourant la littérature sur d'autres pays comme l'Ukraine, la Slovénie ou l'Albanie (Morokvasic et Roudolph 1996, Verbole 2000, Nicholson 2005, Pribytkova 2005), j'ai retrouvé cette frénésie de construire des maisons mais il me semble qu'au Maramureş (de même qu'au pays d'Oaş, décrit par Dana Diminescu ; voir article de D. Moisa dans ce numéro) elle est particulièrement démesurée. Les migrants investissent rarement dans d'autres activités : des petites fabriques, boutiques ou ateliers, etc. La seule utilité évidente de ces nouvelles maisons reste le tourisme et, en attendant leurs propriétaires, elles sont transformées en auberges.

\section{L’élément de la latinité}

L'orientation des migrants prioritairement vers des pays de langue latine (la France ou la Belgique dans les années 1990, l'Italie et l'Espagne ces dernières années), a été justifiée par le même argument : la facilité d'adaptation dans ces endroits "d'esprit latin", semblables quelque part à la Roumanie, la langue ayant son appui dans l'histoire. L'hypothèse de la latinité figurait comme un leitmotiv dans tous les témoignages. C'est par après que je me suis rendue compte que ce discours était celui des media et qu'il masquait, comme le dit Dana Diminescu, une autre réalité, notamment l'épuisement successif de tous les marchés du travail en Europe, en commençant par les plus proches pour aller vers les plus lointains et aussi les plus récemment attirants (Diminescu 1998). Les gens de Maramureş ont été parmi les premiers à partir systématiquement après les années 1990, de la même manière que la migration interne de travail, d'abord en proximité et ensuite de plus en plus loin. Ils sont probablement d'avis que la raison de la latinité tellement présente dans la presse est plus intéressante. Le fait qu'ils n'ont pas de motivation intrinsèque pour soutenir l'argument latin ne prouve pas une cristallisation de l'identité ; autrement dit, le fait qu'ils présentent une version qui n'est pas vraie ne veut pas nécessairement dire qu'ils croient à ce qu'ils disent. Il faut voir les différentes causes et surtout le coté économique qu'on a tendance à cacher.

Les deux derniers pays visés par la migration de travail roumaine sont l'Italie et l'Espagne. Depuis quelques années il y a également un 
important flux de touristes italiens et espagnols. Les hôtes ont souvent l'impression que ces touristes viennent avec un certain mépris pour les Roumains :

les Espagnols ont des préjugés mais moins de mépris que les Italiens. Ils viennent ici en prenant des risques, ils se font une image des Roumains qu'ils voient là-bas, ils n'ont aucune idée de ce qu'ils vont. trouver. Ils finissent toujours par aimer (P.I., f, 42).

Si en 2003 la majorité des touristes étaient francophones, en 2005. 2006, les auberges que j'ai inclues dans ma recherche ont reçu en moyenne : deux familles d'Italiens, une famille d'Espagnols, une de Français et une de Roumains. Ce que j'ai trouvé intéressant, c'est que les hôtes à leur tour considéraient que les Italiens et les Espagnols étaient assez ignorants: "ils ne sont guère si bien informés et documentés d'avance que les Français» (I.G., m, 49). Tout cela pour dire que cette nouvelle vague d'Italiens et d'Espagnols n'est pas indépendante de la massification migratoire des Roumains vers ces deux pays.

\section{Conclusion}

Dans cet article, j'ai essayé de faire la liaison entre deux formes de mobilité, tourisme rural et migration de travail, à travers cinq points d'articulation.

Une spécificité du tourisme rural au Maramureş est que celui-ci est enchâssé dans un contexte économique, social, culturel et historique particulier. L'évolution intéressante du tourisme basé sur l'authenticité de la région est le résultat de la rencontre complexe de différentes activités des multiples acteurs dans la région.

Une certaine "tradition" dans la migration de travail et dans la pluriactivité complexe des gens en tant que stratégie de vie est spécifique à la région, conférant une continuité à un phénomène autrement nouveau dans l'Europe postcommuniste. On se retrouve donc au Maramureş devant deux types de "couloirs de mobilité ": vers et à partir de la région. Ces couloirs se superposent au niveau des pratiques tout comme au niveau des réseaux et des ressources. Les quatre points plus concrets que nous avons abordés ici sont la circulation de l'argent, le rôle des médiateurs culturels des migrants, la question du confort et la façon d'investir dans les maisons, et la problématique de la latinité. 
Les mobilités de toutes sortes sont à la fois les causes et les conséquences d'une diffusion générale des modèles culturels et économiques et les gens sont exposés à un changement social rapide. "La culture locale est une arène pour des choix, des justifications et des représentations conscientes de toutes sortes de voyageurs ou d'indigènes, ces dernières s'adressant souvent à des publics multiples" (Appadurai 2005: 85). Les relations et les comportements se construisent à partir de raisonnements différents de ceux économiques. Dans chacune des situations, la mobilité met l'accent sur les ressources disponibles et développe une certaine expertise en ouvrant la voie vers de nouveaux projets et stratégies de vie, et les individus doivent ainsi vivre au rythme plus vif de l'improvisation. 


\section{Références}

Abram, S., J. Waldren et D. Macleod (dir.), Tourists and Tourism. Identifying with People and Places. Oxford, Berg.

Appadurai, A., 2005 Après le colonialisme. Les conséquences culturelles de la globalisation. Paris, Payot et Rivages.

Boissevain, J., 1977, "Tourism and Development in Malta". Development and Change $8: 523-538$.

Braunlein, P.J. et Lauser, 1997, Ethnologie der migration. Bremen, KeaEdition.

Chapman, M. et R. M. Prothero, 1985, Circulation in Third World Countries. Boston, Routledge.

Crick, M., 1989, "Representations of International Tourism in the Social Sciences. Sun, Sex, Sights, Savings and Servility ». Annual Review of Anthropology $18:$ 307-344.

Diminescu, D., 1998, "Settlement in the Mobility. The Migration to France of Romanian Peasants from the Oas Region. Social Usage of an Economic Handicap or Economic Usage of a Social Handicap?». Conférence de la SSHA, Chicago.

-, R. Ohlinger et V. Rey, 2003, "La construction de l'Europe par ses marges. Stratégies et stratagèmes de la circulation migratoire des Roumains ». Cahiers de recherches de la MIRE 15: 61-69.

Doquet, A., 2001, "Des masques dogon à la dynamique actuelle des identités ». Actualités de la recherche au Mali 3.

Garabuau, I., 1996, « Pour cerner la notion d'authentique ». Anthroepotes I (4).

Lagrave, R. et D. Diminescu, 1999, "Pour une anthropologie des migrations roumaines en France. Le cas du Pays d'Oas ». Migrations Études 91 .

Latour, B., 2006, Changer de société. Refaire de la sociologie. Paris : Éditions de la Découverte.

Lenz, R., 2006, Challenging and Confirming Touristic Representations of the Mediterranean. Migrant Workers in Crete. Oxford, Ramses Working Papers.

Mihailescu, V. 2003, "Omul locului. Ideologie autohtonista în cultura româna ». Dans O. Groza (dir.), Teritorii, scrieri si descrieri: 167-212. Bucarest, Paideia.

Morokvasic M. et H. Roudolph, 1996, Migrants. Les nouvelles mobilités en Europe. Paris, L'Harmattan. 
Nicholson, B., 2005, "Migrants as Agents of Development. Albanian Return Migrants and Micro-Enterprise ". Dans D. Pop. (dir.), New Patterns of Labour Migration in Central and Eastern Europe: 94-110. Cluj-Napoca, Public Policy Center.

O'Reilly, Karen, 2003, "When is a Tourist? The Articulation of Tourism and Migration in Spain's Costa del Sol ». Tourist Studies 3 (3) : 301 317.

2006, "New Forms of Mobility between Migration and Tourism ». Conférence Travel, Tourism and Migration, Université Paris Dauphine et Université Paris 13, 1-2 juin.

Pribytkova, I., 2005, "Labour Market of Ukraine and its Migration Potential in Social Dimension ". Dans D. Pop. (dir.), New Patterns of Labour Migration in Central and Eastern Europe. Cluj-Napoca, Public Policy Center.

Rey, V., O. Groza, I. Ianosi et M. Patroescu, 2002, Atlasul Romaniei. Bucarest, Editions Rao.

Romhild, R., 2003, Practiced Imagination. Tracing Transnational Networks in Crete and Beyond. Research Group on Transnationalism, Working Paper no. 3.

Rosenow, J.E. et G.L. Pulsipher, 1979, Tourism. The Good, the Bad and the Ugly. Lincoln, Century Three.

Stahl, H. H., 1983, Eseuri critice. Despre cultura populara romanesca. Bucarest, Minerva.

Urry, J., 2000, Sociology Beyond Societies. Mobilities for the Twenty-First Century. Londres, Routledge.

Verbole, A., 2000, "Actors, Discourses and Interfaces in the Rural Tourism Development at the Local Community Level in Slovenia. Social and Political Dimensions of the Rural Tourism Development Process ". Journal of Sustainable Tourism 8-6.

Williams, A. M. et C.M. Hall, 2002, « Tourism, Migration, Circulation and Mobility. The Contingencies of Time and Place ». Dans Michael Hall et Allan Williams (dir.), Tourism and Migration. New Relationships between Production and Consumption : 1-60. Londres, Kluwer Academic Publishers. 